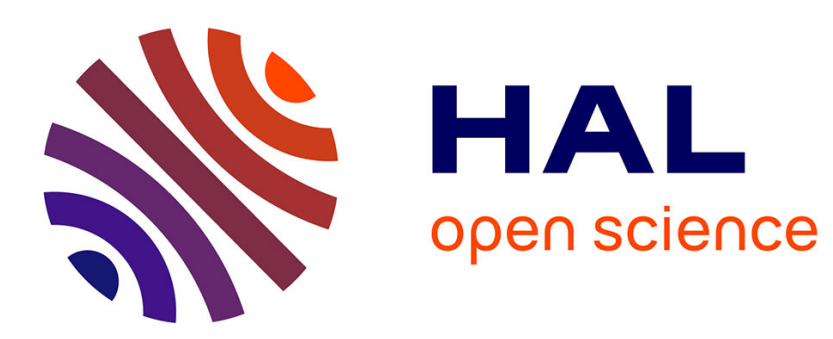

\title{
Cyclone-anticyclone asymmetry and alignment statistics in homogeneous rotating turbulence
}

\author{
Aurore Naso
}

\section{To cite this version:}

Aurore Naso. Cyclone-anticyclone asymmetry and alignment statistics in homogeneous rotating turbulence. Physics of Fluids, 2015, 27, pp.35108. 10.1063/1.4914176 . hal-01298316

\section{HAL Id: hal-01298316 \\ https://hal.science/hal-01298316}

Submitted on 6 Apr 2016

HAL is a multi-disciplinary open access archive for the deposit and dissemination of scientific research documents, whether they are published or not. The documents may come from teaching and research institutions in France or abroad, or from public or private research centers.
L'archive ouverte pluridisciplinaire HAL, est destinée au dépôt et à la diffusion de documents scientifiques de niveau recherche, publiés ou non, émanant des établissements d'enseignement et de recherche français ou étrangers, des laboratoires publics ou privés. 


\title{
Cyclone-anticyclone asymmetry and alignment statistics in homogeneous rotating turbulence
}

\author{
Aurore Naso \\ Laboratoire de Mécanique des Fluides et d'Acoustique, CNRS (UMR 5509), École Centrale \\ de Lyon, Université Claude Bernard Lyon 1 and INSA de Lyon, 36 Avenue Guy de Collongue, \\ F-69134 Écully Cedex, France
}

(Received 27 October 2014; accepted 25 February 2015; published online 16 March 2015)

\begin{abstract}
The cyclone-anticyclone asymmetry occurring in rotating turbulence is investigated through the analysis of the alignment statistics between vorticity and the rotation vector. The advantage of this approach, as compared to the usual measurement of the vertical vorticity skewness, is that the symmetry-breaking can be thus quantified through the analysis of first- and second-order moments, whose statistics convergence is more easily achieved than that of third-order ones. The vorticity/rotation alignment statistics are investigated by direct numerical simulation, both in forced and in freely decaying homogeneous turbulence. In the forced case, the cyclone-anticyclone asymmetry gets stronger as the Rossby number is decreased, whereas the opposite behavior occurs in the decaying case. These findings are shown to be consistent with the existence of a non-monotonic Rossby-number dependence of the asymmetry. A preferential antialignment of vorticity with the rotation vector is found in all the flows considered, a behavior supported by geometrical arguments and by a Taylor expansion of the Navier-Stokes equations for early times of rotation and in the weak rotation limit. A multiscale analysis of the alignment properties between vorticity and the rotation vector is also carried out in the forced case, evidencing the existence of a scale at which both the symmetry-breaking and the collinearity between the two vectors are maximal. ( 2015 AIP Publishing LLC. [http://dx.doi.org/10.1063/1.4914176]
\end{abstract}

\section{INTRODUCTION}

Turbulence subjected to rotation has received considerable interest due to its ubiquity in natural (both astrophysical and geophysical) and industrial flows (e.g., turbomachinery). In these systems, the Coriolis force induced by rotation modifies the turbulence dynamics by imposing a preferred direction, thereby imposing a strong anisotropy in the flow. In turn, this anisotropy results in three generic features of rotating turbulent flows (for a review of the recent experimental and numerical advances made in this field, see, e.g., Ref. 1): it (i) partially inhibits the energy cascade by strongly reducing the dissipation rate, (ii) induces a partial two-dimensionalization of the turbulent velocity field, and (iii) leads to an asymmetry in the vertical (i.e., along the rotation axis) vorticity distribution, with an enhanced probability for large cyclonic vorticity values $(\omega \cdot \boldsymbol{\Omega}>0$, where $\boldsymbol{\Omega}$ is the rotation vector). This latter feature results in a predominance of cyclones over anticyclones, visible in rotating turbulent flows, and is therefore widely referred to as cyclone-anticyclone asymmetry.

Two kinds of arguments have been proposed for explaining this symmetry-breaking. The first one was proposed in Ref. 2: using the Rayleigh's stability criterion, the authors showed that, in the presence of a background rotation, axisymmetric columnar vortices are unstable in regions such that $\omega_{3}<-2 \Omega$, where $\omega_{3} \equiv \omega \cdot \mathbf{e}_{3}$ and $\mathbf{e}_{3} \equiv \mathbf{\Omega} /|\boldsymbol{\Omega}|$ (a convention that will be used throughout the paper). As a consequence, counter-rotating vortices are more likely to be unstable than co-rotating ones if the Rossby number $R o \sim 1$. Other authors showed that Stuart vortices (an exact solution of the steady two-dimensional Euler equations used as a model of the free shear layer ${ }^{3}$ ) were 
destabilized by anticyclonic rotation through a combination of the elliptical and centrifugal instabilities. ${ }^{4}$ Assuming that these stability arguments are relevant in rotating turbulence, they mean that both cyclones and anticyclones form in this type of flow, but that only the former can give rise to long-lived structures at $R o \sim 1$. An alternative argument was developed in Ref. 5: the authors considered a fully developed homogeneous and isotropic turbulent flow instantaneously subjected to a background rotation at $t=0$. Neglecting the induced Poincare force and linearizing the Navier-Stokes equations, they showed that, at $t=0^{+}, \partial\left\langle\omega_{3}^{3}\right\rangle / \partial t=\left.0.4 \Omega\left\langle\omega_{i} s_{i j} \omega_{j}\right\rangle\right|_{t=0}$, where $s_{i j}$ is the rate of strain tensor, and the right-hand side is therefore proportional to the enstrophy production, a quantity widely known to be positive in isotropic turbulence. ${ }^{6}$ According to this analysis, the vertical vorticity skewness is therefore expected to grow from 0 at $t=0^{-}$to a positive value at $t=0^{+}$, a feature taken to be indicative of the dominance of cyclones over anticyclones. This analysis was extended using rapid distortion theory.

In the last decades, many studies have also been devoted to the characterization of the cycloneanticyclone asymmetry. This symmetry-breaking was first visualized in inhomogeneous and forced rotating turbulence, ${ }^{8}$ then investigated quantitatively in experimental and numerical systems, both in freely decaying ${ }^{2,7,9-12}$ and in forced ${ }^{13}$ turbulence. All these studies characterized the cycloneanticyclone asymmetry through the measurement of the skewness of the vertical vorticity component $\omega_{3}$, which vanishes in isotropic turbulence and is positive in rotating flows. More recently, this asymmetry was quantified in statistically stationary flows by using a set of third-order two-point velocity correlation functions, ${ }^{14}$ or the skewness of the azimuthal velocity increments. ${ }^{15}$

We use here another method, based on the investigation of the alignment properties between vorticity and the rotation vector. The analysis of relative orientation between coupled vectors has, indeed, already proven useful for the characterization of turbulence in a wide variety of contexts. In homogeneous and isotropic turbulence (HIT), for instance, the alignment properties of vorticity with the strain eigenvectors or, equivalently, with the vortex stretching vector, turned out to be one of the rare quantitative statistical manifestations of the existence of internal organization in the flow ${ }^{16,17}$ (an analysis that can be extended at various scales, see, e.g., Ref. 18). The nonlinearity depletion phenomenon, another important feature of turbulence occurring at high Reynolds numbers, both in HIT $^{19,20}$ and in more realistic flows, ${ }^{21}$ can also be evidenced by investigating the velocity and vorticity vectors relative orientation. The depletion of nonlinear interaction between Alfvén modes in magnetohydrodynamics ${ }^{22}$ and the self-organization of rotating shear-stratified turbulence ${ }^{23}$ can be cited as other instances of complex features on which insight can be gained through the investigation of alignment statistics.

Motivated by these investigations, we will focus in the present communication on the alignment statistics between vorticity $\omega$ and the rotation axis in homogeneous rotating turbulence. Direct numerical simulations, both in the freely decaying and in the forced cases, will be carried out to this end. In virtue of the aforementioned features of turbulence subjected to a background rotation, it might be expected: (i) that both vectors should be preferentially collinear, a property related to the tendency of the flow to form intense vertical columnar vortices; (ii) that the probability distribution function (PDF) of the cosine between $\boldsymbol{\omega}$ and $\boldsymbol{\Omega}$ is not symmetric, a highly probable signature of the cyclone-anticyclone asymmetry. To the best of our knowledge, this approach has, surprisingly, never been used to characterize the symmetry-breaking of rotating turbulence.

The manuscript is organized as follows. We first describe the numerical algorithm and give the physical parameters of our simulations (Sec. II). The alignment statistics between vorticity and the rotation vector are then presented in Sec. III: the statistics of $\cos (\omega, \boldsymbol{\Omega})$ are first investigated in freely decaying (see Sec. III A) and in forced (see Sec. III B) turbulence, then a multiscale analysis in the latter case is provided in Sec. III C. These numerical results are reinforced in Sec. IV, both by geometric arguments (see Sec. IV A) and through the linearization of the Navier-Stokes equations in the limit of weak rotation (see Sec. IV B). Section V finally contains our concluding remarks. 


\section{NUMERICAL METHOD AND PARAMETERS}

We consider the incompressible forced Navier-Stokes equations describing the dynamics of the velocity field $\mathbf{u}(\mathbf{x}, t)$ in a rotating frame:

$$
\begin{aligned}
\frac{\partial \mathbf{u}}{\partial t}+(\omega+2 \boldsymbol{\Omega}) & \times \mathbf{u}=-\frac{1}{\rho} \nabla p+v \nabla^{2} \mathbf{u}+\mathbf{F}, \\
\nabla \cdot \mathbf{u} & =0,
\end{aligned}
$$

where $\omega=\nabla \times \mathbf{u}(\mathbf{x}, t)$ is the fluctuating vorticity, $p(\mathbf{x}, t)$ is the pressure field (including the centrifugal force contribution), $\rho$ and $v$, respectively, denote the fluid density and kinematic viscosity, and $\mathbf{F}(\mathbf{x}, t)$ is a forcing allowing to maintain a constant level of energy at large scale. Without loss of generality, the frame is chosen in such a way that $\boldsymbol{\Omega}=\boldsymbol{\Omega e}_{3}$, with $\Omega>0$. Equations (1) and (2) are integrated numerically in a periodic box of size $(2 \pi)^{3}$, by using a standard pseudospectral method ${ }^{7,24,25}$ with a resolution of $512^{3}$ collocation points. The advection term is written in a semi-conservative way, and the viscous term is integrated implicitly. Time marching is achieved by a third-order Adams-Bashforth scheme.

The forcing term, tuned to zero in the freely decaying case, is implemented by using the following method. The low wavenumber modes $(|\mathbf{k}| \leq K=1.5)$ are solutions of the Euler equation in the rotating frame, truncated on the sphere $|\mathbf{k}| \leq K .{ }^{26}$ This procedure, already used in HIT $^{27}$ and more recently in rotating turbulence, ${ }^{28}$ maintains a constant amount of energy at the largest scales and allows to reach a statistically steady regime, starting from an incompressible random velocity field. Typical snapshots of the resulting flow are shown in Ref. 28. It is worth mentioning that this forcing is non helical and does not impose any large scale structure in the flow.

As already mentioned, two situations will be considered thereafter. We will first present some results obtained in freely decaying turbulence (Runs D1 and D2). In this configuration, a fully developed isotropic turbulent state was first generated, then the angular velocity $\Omega$ was tuned to a finite value at $t=0$. Ensemble averages $\langle\ldots\rangle$ were approximated by a spatial average taken over the entire computational domain. The level of anisotropy of the turbulence can be quantified by largeand small-scale Rossby numbers, respectively, defined as $R o^{(L)}=u_{r m s} /(L \Omega)$ and $R o^{(\omega)}=\omega_{r m s} / \Omega$, where $u_{r m s} \equiv \sqrt{\left\langle\mathbf{u}^{2}\right\rangle / 3}, \omega_{r m s} \equiv \sqrt{\left\langle\omega^{2}\right\rangle / 3}$, and the integral scale $L \equiv 2 L_{c o r r}$, with $L_{c o r r}$ defined as the correlation lengthscale of the Eulerian velocity field: $L_{c o r r} \equiv \pi /\left(2 u_{r m s}^{2}\right) \int_{0}^{+\infty} d k E(k) / k, E(k)$ denoting the energy spectrum.

The time dependence of $R o^{(L)}$ and $R o^{(\omega)}$ in the freely decaying simulations is shown in Fig. 1. Naturally, both quantities decay monotonically, and $R o^{(L)}<R o^{(\omega)}$, indicating, respectively, that the effects of rotation get stronger as time increases and that they are more pronounced at large scales.

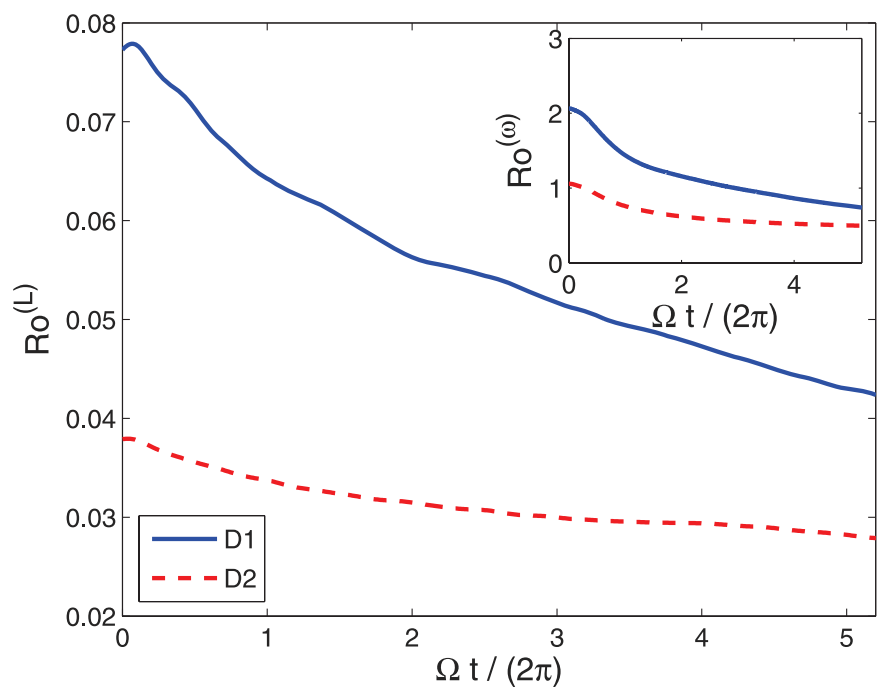

FIG. 1. Time dependence of the large- and small-scale Rossby numbers in Runs D1 and D2. 
TABLE I. Physical parameters of Runs F1 to F3 (forced turbulence).

\begin{tabular}{lccccc}
\hline \hline Run & $R o^{(L)}$ & $R o^{(\omega)}$ & $L / \ell_{Z}$ & $\ell_{Z} / \eta$ & $R_{\lambda}$ \\
\hline F1 & 0.18 & 4.3 & 16 & 19 & 240 \\
F2 & 0.12 & 2.5 & 34 & 7 & 280 \\
F3 & 0.07 & 1.1 & 88 & 3 & 420 \\
\hline
\end{tabular}

These are well-known characteristics of decaying rotating turbulence. $R o^{(L)} \ll 1$ throughout the calculations, meaning that, at large scales, background rotation effects are dominant over nonlinear ones at any time. At the largest times of the calculations, $R o^{(\omega)}$ is also $<1$ in both runs, thereby indicating that rotation effects then dominate at all scales. In both runs, the Taylor Reynolds number $R_{\lambda}$ varies from $\approx 200$ at $t=0$ to $\approx 280$ at the end of the calculation $(\Omega t /(2 \pi) \approx 5.2)$.

Simulations in the forced case were also carried out (Runs F1 to F3). Anisotropy of the resulting flows can be characterized by the Rossby numbers, and by the Zeman scale $\ell_{Z} \equiv\left(\varepsilon / \Omega^{3}\right)^{1 / 2}$ (where $\varepsilon$ is the energy dissipation rate), ${ }^{29}$ which quantifies the ratio between nonlinearity and rotation effects and was found to give a reasonable threshold between anisotropic $\left(r>\ell_{Z}\right)$ and isotropic $\left(r<\ell_{Z}\right)$ scales. ${ }^{30,31}$ The ratios between $\ell_{Z}$ and $L$ and $\eta$ (Kolmogorov scale), as well as $R o^{(L)}, R o^{(\omega)}$, and $R_{\lambda}$ (Taylor Reynolds number), are gathered in Table I. In these simulations, ensemble averages were approximated by spatial and temporal averages.

\section{ALIGNMENT STATISTICS BETWEEN VORTICITY AND THE ROTATION VECTOR}

\section{A. Statistics of the relative orientation between $\omega$ and $\Omega$ in decaying turbulence}

We first investigate the alignment statistics between $\omega$ and $\boldsymbol{\Omega}$ in decaying turbulence (Runs D1 and D2). Figure 2 shows the time dependence of the low order moments of the cosine of the angle between the two vectors. As expected, at $t=0$, when turbulence is still isotropic, the values of the three quantities are those that one would expect for two uncorrelated vectors, i.e., the odd moments are zero and the variance is equal to $1 / 3$. In the early times of the turbulence decay, the three quantities deviate monotonously from their isotropic values. In particular, $\langle\cos (\boldsymbol{\omega}, \mathbf{\Omega})\rangle$ is a decreasing function of time, thus becoming negative. This nonzero value naturally reflects the cyclone-anticyclone asymmetry. However, its sign is rather counterintuitive: it means that, in

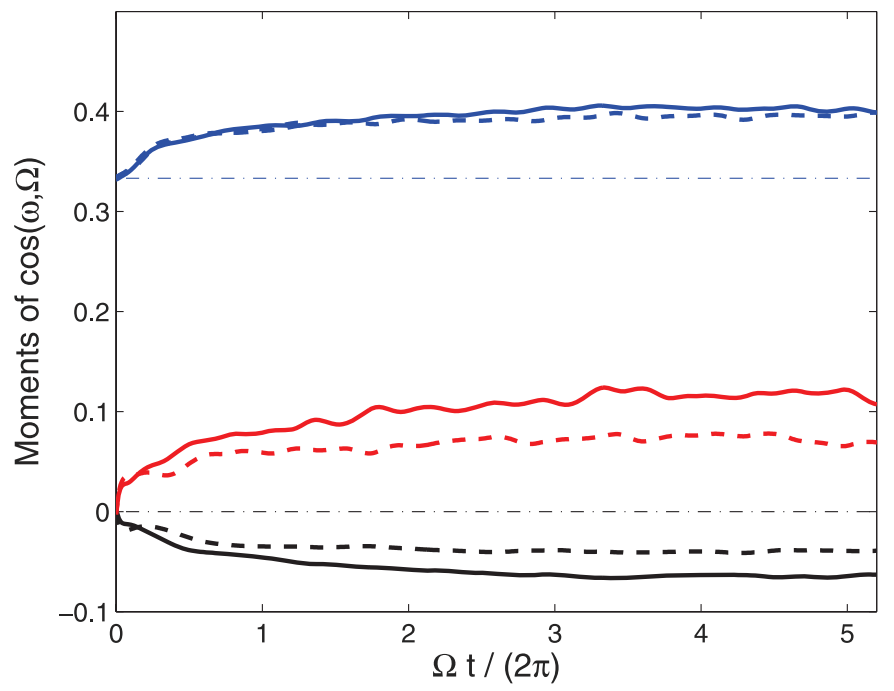

FIG. 2. Low order moments of $\cos (\omega, \boldsymbol{\Omega})$ vs time, in Runs D1 and D2: mean $\langle X\rangle$ (black), variance $\left\langle(X-\langle X\rangle)^{2}\right\rangle$ (blue), skewness $\left\langle(X-\langle X\rangle)^{3}\right\rangle /\left\langle(X-\langle X\rangle)^{2}\right\rangle^{3 / 2}$ (red), with $X \equiv \cos (\boldsymbol{\omega}, \boldsymbol{\Omega})$. Solid line: run D1; dashed line: run D2. The values of these moments in HIT are indicated by the dash-dotted lines. 


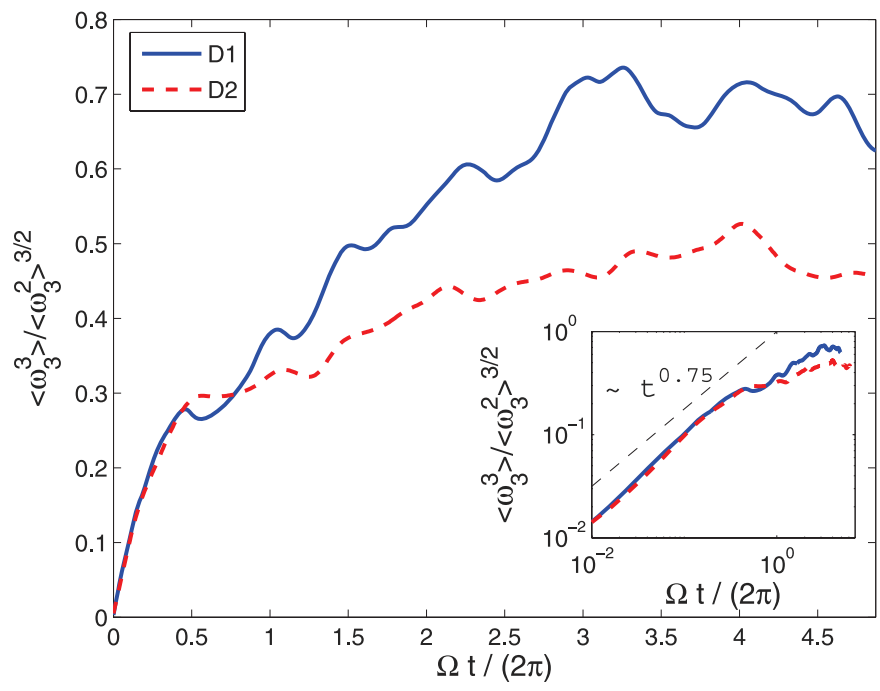

FIG. 3. Skewness of $\omega_{3}$ vs time, in Runs D1 and D2 $\left(\left\langle\omega_{3}\right\rangle=0\right.$ by homogeneity).

average, vorticity is preferentially oriented in a direction opposite to that of $\boldsymbol{\Omega}$. This observation will be interpreted in Subsection IV A.

As shown in Fig. 2, during the first stage of turbulence decay, the variance and skewness of $\cos (\omega, \boldsymbol{\Omega})$ are increasing functions of time (up to some spurious time fluctuations especially visible in the skewness signal, that can be explained by the fact that the convergence of this third-order quantity is probably not perfectly achieved when ensemble average is approximated by a spatial one). As a consequence, in these decaying homogeneous rotating flows, the skewness of $\cos (\omega, \boldsymbol{\Omega})$ is found to become positive, and its variance to be $>1 / 3$.

For $\Omega t /(2 \pi) \gtrsim 3$, the three moments oscillate around constant values, thereby indicating a steady cyclone-anticyclone asymmetry. The deviation of these quantities from their isotropic values is the largest in Run D1, in which the Rossby numbers are higher than in Run D2: the symmetrybreaking is therefore stronger in the flow in which the ratio between rotation effects and nonlinear ones is the smallest. As a comparison, we show in Fig. 3 the time dependence of the vertical vorticity skewness in both runs. This quantity also reaches plateaus at $\Omega t /(2 \pi) \approx 3$, after a transient growth during which it scales as $t^{0.75}$, two features already observed in previous studies of decaying rotating turbulence. ${ }^{2,7,10}$ Similarly to the moments of $\cos (\omega, \boldsymbol{\Omega})$, the skewness of $\omega_{3}$ is the largest in Run D1 (in which the Rossby numbers are the largest). ${ }^{7}$ In the early times of turbulence decay, no clear scaling of the moments of $\cos (\omega, \boldsymbol{\Omega})$ can be evidenced.

\section{B. Statistics of the relative orientation between $\omega$ and $\Omega$ in forced turbulence}

We now turn to the measurement of the statistics of $\cos (\omega, \boldsymbol{\Omega})$ in forced rotating turbulence. This will allow us to accumulate more statistics for each value of the Rossby number and to investigate the $R o$ dependence of the results in these statistically stationary flows.

The mean, variance, and skewness of $\cos (\omega, \boldsymbol{\Omega})$ are plotted as a function of the large scale Rossby number in Fig. 4 (Runs F1 to F3). As expected, they all tend to their values in isotropic turbulence (infinite Rossby numbers) as $R o^{(L)}$ increases. In rotating flows, $\langle\cos (\omega, \boldsymbol{\Omega})\rangle$ is found to be negative. As in freely decaying turbulence, these nonzero values trace the cyclone-anticyclone antisymmetry present in the flow, their sign expresses the fact that vorticity tends to be preferentially oriented in a direction opposite to that of the rotation vector. The variance and skewness are found to be larger than their isotropic values, as was the case in decaying turbulence. However, the deviation of the $\cos (\boldsymbol{\omega}, \boldsymbol{\Omega})$ moments from their isotropic values increases at decreasing $R o^{(L)}$, a behavior opposite to the one observed in decaying turbulence (see Sec. III A). In the range of Rossby numbers considered here, the asymmetry in forced flows is therefore stronger as rotation effects get 


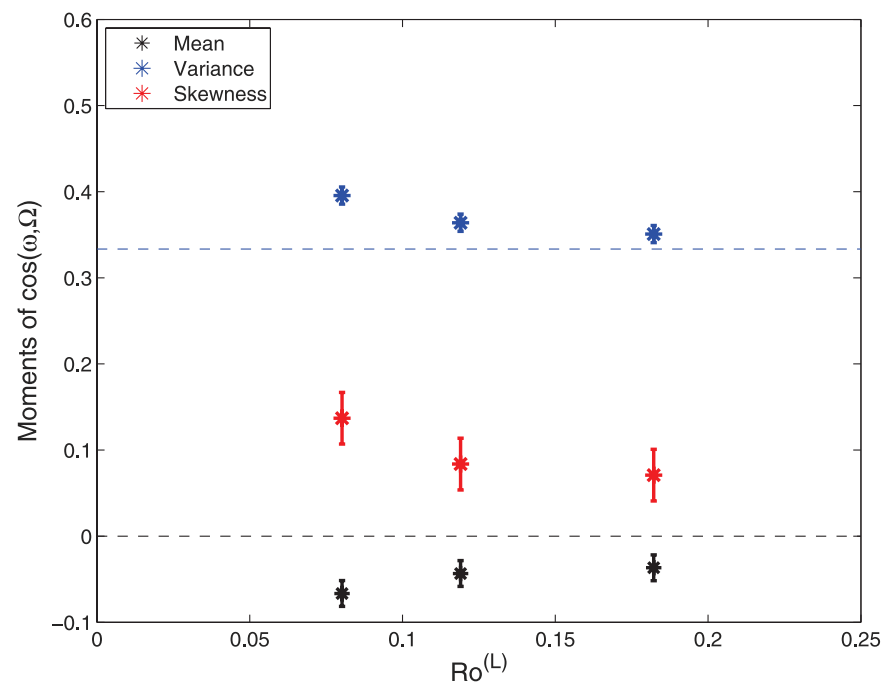

FIG. 4. $R o^{(L)}$ dependence of the $\cos (\boldsymbol{\omega}, \boldsymbol{\Omega})$ mean, variance, and skewness (same definitions as in Fig. 2), in forced turbulence. The horizontal error bars are within the symbols size. The horizontal dashed lines indicate the values of these quantities in HIT (infinite Rossby number).

more and more pronounced as compared to nonlinear ones. This result is confirmed by the measurement of the skewness of vertical vorticity in our simulations, which varies monotonously from $\approx 0.2$ in Run F1 to $\approx 0.8$ in Run F3. Interestingly, this behavior is similar to the one observed, in the same range of parameters, in recent experiments of (inhomogeneous) forced rotating turbulence, ${ }^{14}$ in which the symmetry-breaking was quantified through the measurement both of the vertical vorticity skewness and of velocity correlation functions. The comparison of our results in decaying and in forced flows will be done, and a discussion provided, in Sec. V.

A more refined information can be given by the probability distribution function of $\cos (\omega, \boldsymbol{\Omega})$, plotted in Fig. 5 for different values of the Rossby number (Runs F2 and F3). In the most anisotropic flow (Run F3), the PDF clearly peaks at \pm 1 , indicating that vorticity is preferentially collinear with the rotation axis, a well known feature of rotating turbulence. It also exhibits an asymmetry, with an enhanced probability for negative values that explains the negative sign of $\langle\cos (\omega, \boldsymbol{\Omega})\rangle$. The PDF obtained at higher Rossby number exhibits the same properties, although less pronounced, and is therefore closer to the flat distribution expected in isotropic turbulence. This confirms that, in

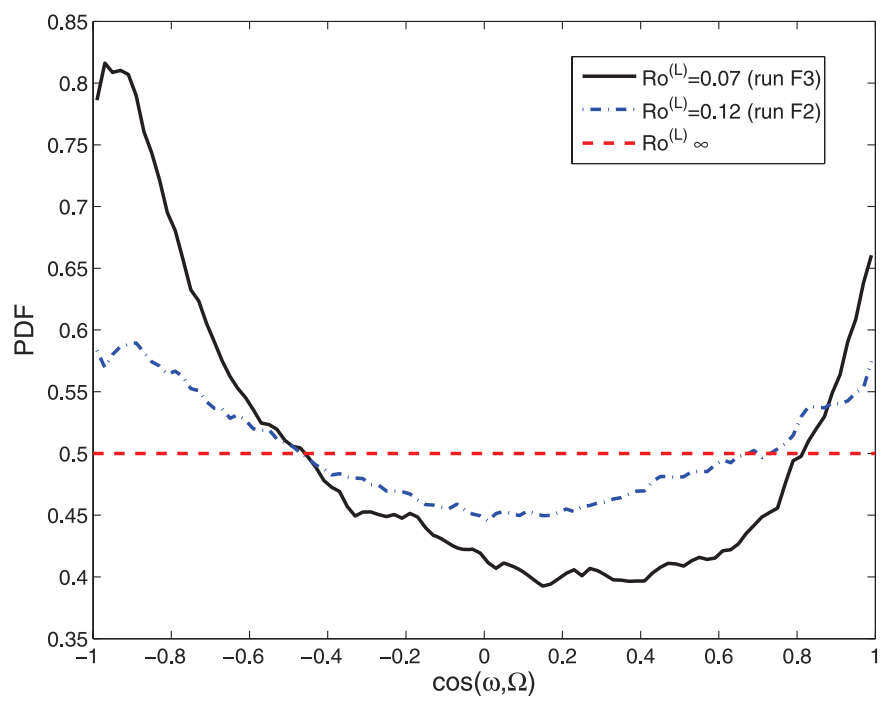

FIG. 5. PDF of $\cos (\omega, \boldsymbol{\Omega})$ for different Rossby numbers, in forced turbulence. 
the range of Rossby numbers considered here, the cyclone-anticyclone asymmetry is stronger for smaller $R o^{(L)}$.

\section{Multiscale analysis in forced turbulence}

The scale dependence of the cyclone-anticyclone asymmetry, in the framework of alignment properties between vorticity and the rotation vector, is now addressed. To do so, one needs to consider a generalized vorticity vector accounting for the flow variations at a given scale. A suitable approach, to this end, consists in using the "perceived" vorticity, constructed from the antisymmetric part of the velocity gradient tensor defined by interpolation from the locations and velocities of a set of four points. This approach was introduced in the framework of Lagrangian turbulence (in that case, the points are fluid particles), and allowed to investigate the structure of isotropic turbulence at different scales. ${ }^{18,32-34}$ We perform here Eulerian measurements, by considering sets of four points equally spaced in the flow to construct a perceived velocity gradient tensor, a procedure already used in a different context in rotating turbulence. ${ }^{28}$ Several distances $r_{0}$ between the points, ranging from $\approx \eta$ to $\approx L / \sqrt{2}$, were considered, so as to cover the entire inertial range. Between $2 \times 10^{6}$ and $4 \times 10^{6}$ randomly oriented tetrads were considered in each flow and for each value of $r_{0}$, in order to ensure a satisfactory convergence of the statistics.

The mean, variance, and skewness of the cosine between the perceived vorticity $\tilde{\boldsymbol{\omega}}\left(r_{0}\right)$ and $\boldsymbol{\Omega}$ are plotted, in Fig. 6, as a function of scale. For the smallest values of $r_{0}$, the alignment properties between the usual vorticity and $\boldsymbol{\Omega}$ are recovered, i.e., (i) the mean of $\cos (\tilde{\boldsymbol{\omega}}, \boldsymbol{\Omega})$ is negative while its variance and skewness are larger than their isotropic values; (ii) the three quantities deviate more significantly from their isotropic reference values as the Rossby number is decreased. These two trends are observed at any scale, meaning in particular that the perceived vorticity is always preferentially antialigned with $\boldsymbol{\Omega}$. All the quantities tend to their isotropic (uncorrelated) values as $r_{0} \rightarrow L$, reflecting the fact that the statistics between the four points become uncorrelated in this limit. But the most remarkable feature is the extremum displayed by all the curves in Fig. 6. It points out the existence of a characteristic scale at which the cyclone-anticyclone asymmetry is maximum. This scale is the same for the three moments investigated here, and much larger than the Zeman scales of the flows (indicated by vertical lines). It seems to depend very weakly on the Rossby number, always being of order $L / 3-L / 2$. Such a characteristic scale of the cyclone-anticyclone asymmetry has been evidenced in experimental studies, in inhomogeneous forced turbulence, through

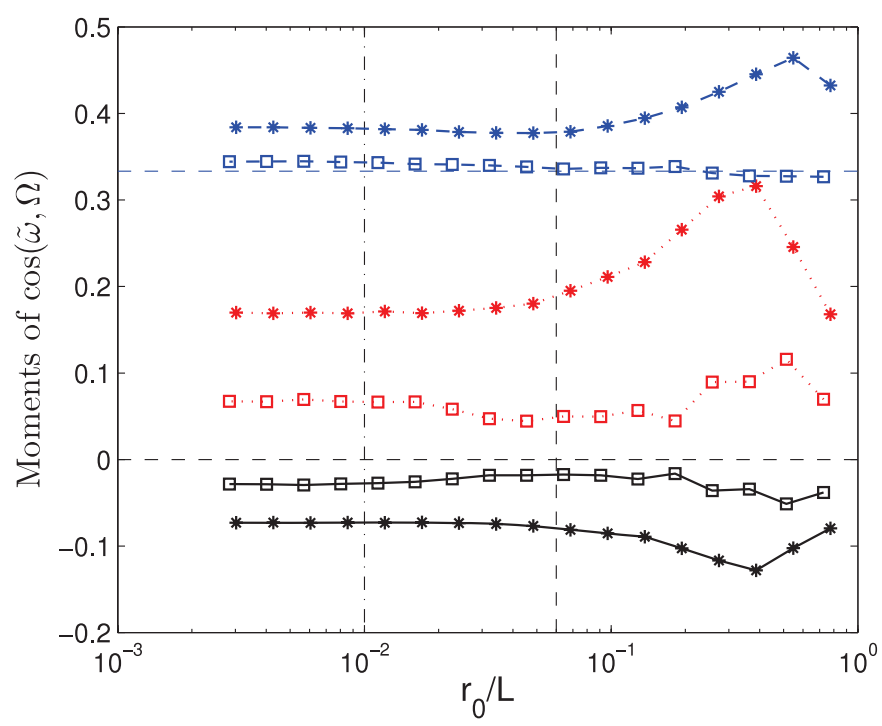

FIG. 6. Scale dependence of the moments of $\cos (\tilde{\boldsymbol{\omega}}, \boldsymbol{\Omega})$, for different Rossby numbers, in forced turbulence. Mean (black solid lines), variance (blue dashed lines), and skewness (red dotted lines). Squares: run F1; stars: run F3. The horizontal (resp. vertical) lines indicate the isotropic values (resp. Zeman scales). 


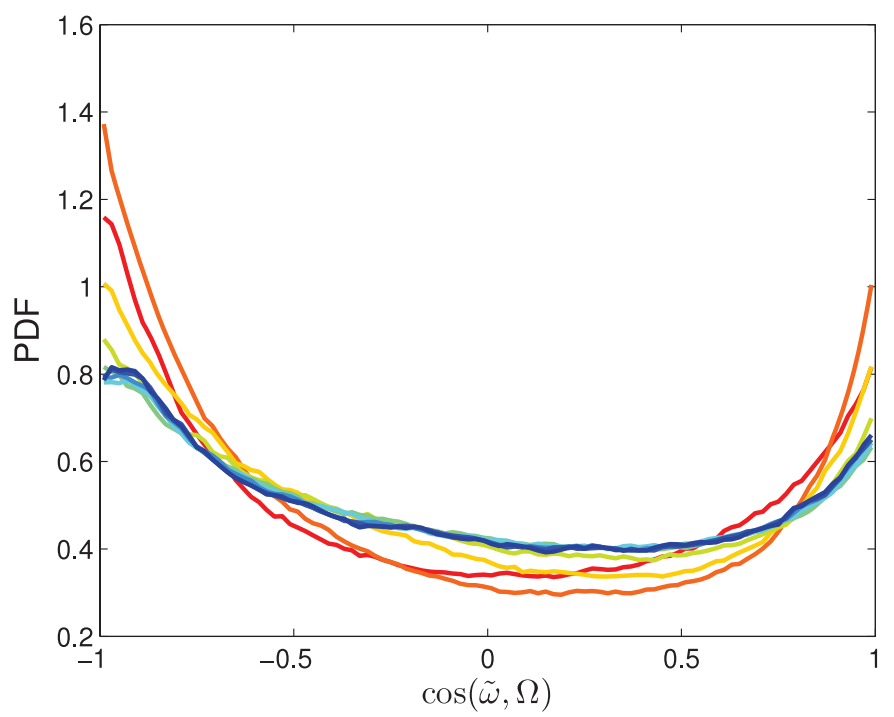

FIG. 7. PDF of $\cos (\tilde{\boldsymbol{\omega}}, \boldsymbol{\Omega})$ for $r_{0} / L=3 \times 10^{-3}, 6 \times 10^{-3}, 1.2 \times 10^{-2}, 2.4 \times 10^{-2}, 4.8 \times 10^{-2}, 9.7 \times 10^{-2}$, $0.19,0.39,0.77$, from blue to red (Run F3).

the investigation of velocity correlation functions,${ }^{14}$ and in the late stage of decaying turbulence, measuring the vertical vorticity skewness. ${ }^{12}$ Our numerical approach not only allows to investigate statistically homogeneous and stationary flows, but also to provide accurate values of the lengthscales characterizing them (Kolmogorov, integral and Zeman scales).

The nontrivial scale dependence of the cyclone-anticyclone asymmetry can also be illustrated by plotting the PDF of $\cos \left(\tilde{\boldsymbol{\omega}}\left(r_{0}\right), \boldsymbol{\Omega}\right)$. This function is shown in Fig. 7 for different values of $r_{0}$, in the most anisotropic flow (Run F3). The PDFs' peaks at \pm 1 confirm that vorticity is preferentially collinear with the rotation vector for any $r_{0}$. This feature, together with the asymmetry of the PDFs that reflects the symmetry-breaking, is the most pronounced at the largest scales. However, the evolution of the PDFs as $r_{0}$ increases is nonmonotonic: the peaks of the dark orange curve $\left(r_{0} / L=0.39\right)$ are, without any ambiguity, more pronounced than those of the red one $\left(r_{0} / L=0.77\right)$. This representation therefore also evidences the existence of a scale, $\approx L / 2-L / 3$, at which the cyclone-anticyclone asymmetry is maximal. It appears furthermore that the collinearity of vorticity and rotation is maximal at this scale.

\section{INTERPRETATION OF THE PREFERENTIAL ANTIALIGNMENT BETWEEN VORTICITY AND THE ROTATION VECTOR}

\section{A. Geometric interpretation}

We now focus on the negative sign of $\langle\cos (\omega, \boldsymbol{\Omega})\rangle$, evidenced at any Rossby number and any scale and reflecting a tendency of vorticity to be aligned in a direction opposite to that of the rotation vector. It is shown here that this counterintuitive result can be explained by simple geometrical arguments. Given that $\cos (\omega, \boldsymbol{\Omega})=\omega_{3} /\left(\omega^{3}\right)^{1 / 2}$ and that $\left\langle\omega_{3}\right\rangle=0$ in homogeneous turbulence, the sign of $\langle\cos (\omega, \boldsymbol{\Omega})\rangle$ can only be explained by comparing the enstrophy statistics conditioned on the sign of $\omega_{3}$. The conditioned averages $\left\langle\omega^{2} \mid \omega_{3}>0\right\rangle$ and $\left\langle\omega^{2} \mid \omega_{3}<0\right\rangle$ are shown, both in decaying and in forced turbulence, in Fig. 8. Not surprisingly, the former is always larger than the latter, thereby indicating that vorticity is, in average, more intense in cyclonic structures than in anticyclonic ones. This is a straightforward consequence of the enhanced probability of positive values of $\omega_{3}$ with respect to negative ones, characteristic of the predominance of cyclones over anticyclones. In Fig. 8, this asymmetry is, once more, shown to get stronger (respectively weaker) as the Rossby number is decreased in the forced (respectively decaying) flows considered in the present investigation.

The explanation of the negative sign of $\langle\cos (\omega, \boldsymbol{\Omega})\rangle$ is now straightforward, as illustrated in Fig. 9. In average, in homogeneous turbulence the projections of cyclonic and anticyclonic vorticity 

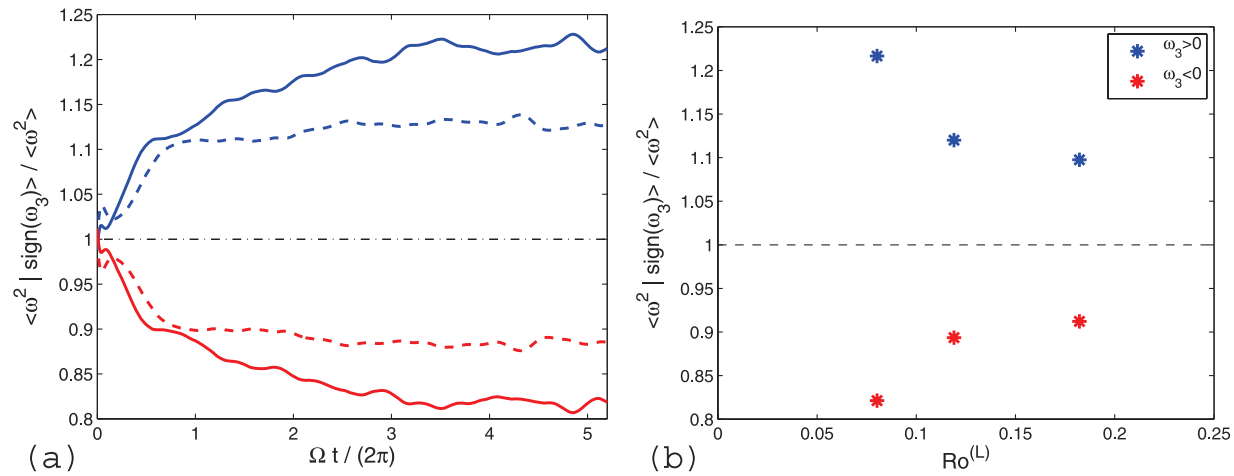

FIG. 8. Mean enstrophy conditioned on the sign of $\omega_{3}\left(\omega_{3}>0\right.$ in blue, $<0$ in red), and normalized by the total enstrophy: (a) vs time, in decaying turbulence (Runs D1 (solid lines) and D2 (dashed lines)); (b) vs $R o^{(L)}$, in forced turbulence.

vectors on the rotation axis are equal (in absolute value), but the magnitudes of the latter are smaller (thereby indicating that enstrophy is more intense in cyclonic structures than in anticyclonic ones). These two properties lead to a stronger collinearity of anticyclonic vorticity with rotation, and therefore to an overall negative $\langle\cos (\omega, \boldsymbol{\Omega})\rangle$.

\section{B. Linearization of the Navier-Stokes equations in the weak rotation limit}

A second argument in favor of the negative sign of $\langle\cos (\omega, \boldsymbol{\Omega})\rangle$ can be obtained by considering the Navier-Stokes equations, assuming that a developed isotropic turbulent flow is suddenly subjected to a background rotation and neglecting the Poincare force. The same kind of analysis was

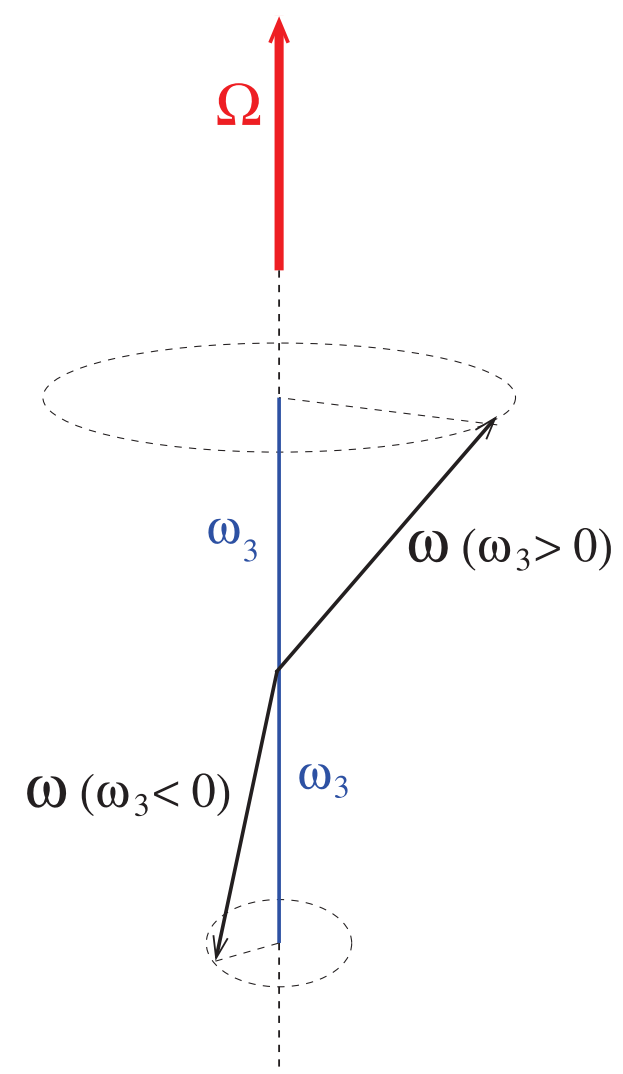

FIG. 9. Schematic illustration of the geometrical mechanisms leading to the preferential antialignment of vorticity with the rotation vector. 
performed in Ref. 5 and gave a theoretical support to the positive skewness of vertical vorticity measured in experiments and numerical simulations.

We start from the equation satisfied by the vorticity components, in the rotating frame, in the absence of forcing

$$
\frac{D \omega_{i}}{D t}=s_{i j} \omega_{j}+2 \Omega s_{i 3}+\left(\omega \times \Omega \mathbf{e}_{3}\right)_{i}+v \partial_{j j} \omega_{i},
$$

where $D / D t$ is the Lagrangian derivative and $s_{i j}=\left(\partial_{i} u_{j}+\partial_{j} u_{i}\right) / 2$ is the rate of strain tensor. The Einstein summation convention is used throughout the derivation. As a consequence,

$$
\frac{1}{2} \frac{D\left(\omega^{2}\right)}{D t}=\omega_{i} s_{i j} \omega_{j}+2 \Omega \omega_{i} s_{i 3}+v \omega_{i} \partial_{j j} \omega_{i},
$$

and

$$
\frac{D \omega_{3}}{D t}=\omega_{i} s_{i 3}+2 \Omega s_{33}+v \partial_{i i} \omega_{3} .
$$

The time derivative of $\cos (\omega, \boldsymbol{\Omega})$ therefore reads:

$$
\begin{aligned}
\frac{D \cos (\boldsymbol{\omega}, \boldsymbol{\Omega})}{D t}= & \frac{1}{\left(\omega^{2}\right)^{3 / 2}}\left[\omega^{2}\left(\omega_{i} s_{i 3}+2 \Omega s_{33}+v \partial_{i i} \omega_{3}\right)-\omega_{3}\left(\omega_{i} s_{i j} \omega_{j}\right.\right. \\
& \left.\left.+2 \Omega \omega_{i} s_{i 3}+v \omega_{i} \partial_{j j} \omega_{i}\right)\right] .
\end{aligned}
$$

Let us assume now that the flow is statistically homogeneous and isotropic at the initial time $t=0$, at which $\Omega$ is instantaneously tuned to a finite value. According to Eq. (6), the contribution of the rotation rate to $D\langle\cos (\omega, \boldsymbol{\Omega})\rangle / D t$ is then $2 \Omega\left\langle s_{33} /\left(\omega^{2}\right)^{1 / 2}-\omega_{i} s_{i 3} \omega_{3} /\left(\omega^{2}\right)^{3 / 2}\right\rangle$, and has the same sign as

$$
\left\langle s_{33} /\left(\omega^{2}\right)^{1 / 2}\right\rangle-\left\langle\omega_{i} s_{i 3} \omega_{3} /\left(\omega^{2}\right)^{3 / 2}\right\rangle .
$$

At $t=0$, statistical isotropy is still satisfied, and the first term of Eq. (7) can therefore be rewritten as $\left\langle s_{i i} /\left(\omega^{2}\right)^{1 / 2}\right\rangle / 3$, which is zero due to the incompressibility condition $s_{i i}=0$. Isotropy imposes that the second term of Eq. (7) is equal to one third of a generalized total enstrophy production rate $\left\langle\omega_{i} s_{i j} \omega_{j} /\left(\omega^{2}\right)^{3 / 2}\right\rangle$, that we have found to be positive in all our simulations of homogeneous and isotropic turbulence. Note that the mean values of the standard enstrophy production $\omega_{i} s_{i j} \omega_{j}$ and of its rate, $\omega_{i} s_{i j} \omega_{j} / \omega^{2}$, are known to be positive in HIT (see, e.g., Ref. 6). As a consequence, at $t=0^{+}$ the quantity (7) is negative, and $\langle\cos (\omega, \boldsymbol{\Omega})\rangle$, that was equal to zero at $t=0^{-}$, is expected to become negative as well.

\section{CONCLUSION AND DISCUSSION}

The alignment statistics between vorticity and the rotation vector were investigated, using a unique numerical setup, in decaying and in forced statistically homogeneous turbulence. Beyond the very well known preferential collinearity of these two vectors, we have shown that the analysis of these statistics provides a detailed characterization of the cyclone-anticyclone asymmetry. Unlike that of the vertical vorticity, the mean of $\cos (\omega, \boldsymbol{\Omega})$ is nonzero. As a consequence, the measurement of its first (and second) moments, whose statistics converge faster than those of third-order quantities, is sufficient to characterize the cyclone-anticyclone asymmetry. The price to pay for this faster convergence is that it necessitates the simultaneous measurement of the three vorticity components, not easily accessible experimentally.

Our results confirm some previous findings according to which, in statistically stationary flows, the cyclone-anticyclone asymmetry gets more pronounced as the Rossby number is decreased, while the opposite behavior occurs in freely decaying turbulence. Interestingly, in the present investigation the Rossby numbers of the decaying runs (in the late stage of the decay) are slightly lower than those of the forced simulations. Our results might therefore indicate that the cycloneanticyclone asymmetry is the strongest in an intermediate range of Rossby numbers: $R o^{(L)} \approx 0.05$; $R o^{(\omega)} \approx 1$. The existence of a value of the Rossby number at which this asymmetry is maximal was 
already evidenced in decaying ${ }^{2,35}$ and in forced ${ }^{15}$ turbulent flows. It is worth mentioning that the value $R o^{(\omega)} \approx 1$ found in our investigation is in agreement with that of Ref. 2 (in which $R o^{(\omega)} \approx 0.8$ with our definition). A straightforward consequence of this is the fact that the asymmetry manifests itself if both rotation and nonlinear effects are strong enough. This result would therefore give more support to the interpretation of the phenomenon based on a preferential stretching of cyclonic vorticity ${ }^{5}$ than to that related to instabilities also observed in laminar flows. ${ }^{2}$

A multiscale analysis of the vorticity/rotation alignment was also performed, evidencing the existence of a scale, much larger than $\ell_{Z}$ and close to $L$, at which both the symmetry-breaking and the collinearity between the two vectors are maximal. One of our main findings is the tendency of $\omega$ to be oriented in a direction opposite to that of $\boldsymbol{\Omega}$, a feature that we have interpreted through the investigation of the enstrophy statistics conditioned on the sign of the vertical vorticity component. These indicate, not surprisingly, that enstrophy is more intense in cyclonic structures than in anticyclonic ones. These statistics incidentally appear as another indicator of the cyclone-anticyclone asymmetry which does not necessitate the measurement of third-order quantities. Our result is also supported by the linearization of the Navier-Stokes equations at small time and in the weak rotation limit, an analysis that could be interestingly extended at longer times through the use of rapid distortion theory. ${ }^{36,37}$

Given that the former argument is partially based on the statistical homogeneity of the flow, it would be interesting to study the same kind of statistics in inhomogeneous flows for which $\left\langle\omega_{3}\right\rangle \neq 0$ (e.g., channel flow), so as to check if the preferential antialignment observed here is robust. The Reynolds numbers in the simulations presented here are moderate $\left(R_{\lambda}<400\right)$, and much information could be certainly brought by studies at larger $R_{\lambda}$. Another line of research would consist in splitting the vorticity field into coherent and uncoherent parts (see, e.g., Ref. 38) and to carry out the statistical analysis of the asymmetry on both sides separately. Finally, we have shown that alignment properties are efficient tools for probing the structure of rotating turbulence. The investigation of other statistics of this type, related to geometrical statistics, will be presented in a forthcoming publication.

\section{ACKNOWLEDGMENTS}

The author acknowledges stimulating discussions with F. Godeferd, C. Cambon and F. Moisy. F. Godeferd is also gratefully thanked for providing the code and for a careful reading of the manuscript. This work has been performed using the HPC resources provided by GENCI-CINES and GENCI-IDRIS (Grant No. x20142a2206).

${ }^{1}$ F. S. Godeferd and F. Moisy, "Structure and dynamics of rotating turbulence: A review of recent experimental and numerical results," Appl. Mech. Rev. 67, 030802 (2015).

${ }^{2}$ P. Bartello, O. Metais, and M. Lesieur, "Coherent structures in rotating three-dimensional turbulence," J. Fluid Mech. 273, 1-29 (1994).

${ }^{3}$ J. T. Stuart, "On finite amplitude oscillations in laminar mixing layers," J. Fluid Mech. 29, 417-440 (1967).

${ }^{4}$ F. S. Godeferd, C. Cambon, and S. Leblanc, "Zonal approach to centrifugal, elliptic and hyperbolic instabilities in Stuart vortices with external rotation,” J. Fluid Mech. 449, 1-37 (2001).

${ }^{5}$ J.-N. Gence and C. Frick, "Birth of the triple correlations of vorticity in an homogeneous turbulence submitted to a solid body rotation," C. R. Acad. Sci. Paris Serie IIB 329, 351-356 (2001).

${ }^{6}$ A. Tsinober, An Informal Conceptual Introduction to Turbulence (Springer, 2009).

${ }^{7}$ L. J. A. van Bokhoven, C. Cambon, L. Liechtenstein, F. S. Godeferd, and H. J. H. Clercx, "Refined vorticity statistics of decaying rotating three-dimensional turbulence," in Particle-Laden Flow: From Geophysical to Kolmogorov Scales, ERCOFTAC Series Vol. 11, pp. 115-127 (Springer Netherlands, 2007).

${ }^{8}$ E. J. Hopfinger, F. K. Browand, and Y. Gagne, "Turbulence and waves in a rotating tank," J. Fluid Mech. 125, 505-534 (1982).

${ }^{9}$ C. Morize, F. Moisy, and M. Rabaud, "Decaying grid-generated turbulence in a rotating tank," Phys. Fluids 17, 095105 (2005).

${ }^{10}$ P. J. Staplehurst, P. A. Davidson, and S. B. Dalziel, "Structure formation in homogeneous freely decaying rotating turbulence," J. Fluid Mech. 598, 81-105 (2008).

11 B. Sreenivasan and P. A. Davidson, "On the formation of cyclones and anticyclones in a rotating fluid," Phys. Fluids 20, 085104 (2008).

${ }^{12}$ F. Moisy, C. Morize, M. Rabaud, and J. Sommeria, "Decay laws, anisotropy and cyclone-anticyclone asymmetry in decaying rotating turbulence," J. Fluid Mech. 666, 5 (2011).

${ }^{13}$ F. S. Godeferd and L. Lollini, "Direct numerical simulations of turbulence with confinement and rotation," J. Fluid Mech. 393, 257 (1999). 
${ }^{14}$ B. Gallet, A. Campagne, P.-P. Cortet, and F. Moisy, "Scale-dependent cyclone-anticyclone asymmetry in a forced rotating turbulence experiment," Phys. Fluids 26, 035108 (2014).

${ }^{15}$ E. Deusebio, G. Boffetta, E. Lindborg, and S. Musacchio, “Dimensional transition in rotating turbulence," Phys. Rev. E 90 , 023005 (2014).

${ }^{16}$ W. T. Ashurst, A. R. Kerstein, R. M. Kerr, and C. H. Gibson, "Alignment of vorticity and scalar gradient with strain rate in simulated Navier-Stokes turbulence," Phys. Fluids 30, 2343 (1987).

${ }^{17}$ M. Kholmyansky, A. Tsinober, and S. Yorish, "Velocity derivatives in the atmospheric surface layer at RelÄ104," Phys Fluids 13, 311 (2001).

${ }^{18}$ A. Pumir, E. Bodenschatz, and H. Xu, "Tetrahedron deformation and alignment of perceived vorticity and strain in a turbulent flow," Phys. Fluids 25, 035101 (2013).

${ }^{19}$ R. H. Kraichnan and R. Panda, "Depression of nonlinearity in decaying isotropic turbulence," Phys. Fluids 31, 2395 (1988).

${ }^{20}$ W. J. T. Bos and R. Rubinstein, "On the strength of the nonlinearity in isotropic turbulence," J. Fluid Mech. 733, 158 (2013).

${ }^{21}$ R. Monchaux, F. Ravelet, B. Dubrulle, A. Chiffaudel, and F. Daviaud, "Properties of steady states in turbulent axisymmetric flows," Phys. Rev. Lett. 96, 124502 (2006).

22 J. C. Perez and S. Boldyrev, "Role of cross-helicity in magnetohydrodynamic turbulence," Phys. Rev. Lett. 102, 025003 (2009).

${ }^{23}$ A. B. Pieri, F. S. Godeferd, C. Cambon, B. Dubrulle, and S. Thalabard, "Cross-helicity in rotating homogeneous shearstratified turbulence," Phys. Rev. Lett. 112, 114501 (2014).

${ }^{24}$ R. Rogallo, "Numerical experiments in homogeneous turbulence," NASA Technical Memorandum 81315, 1981. Available at http://ntrs.nasa.gov/search.jsp?R=19810022965.

${ }^{25}$ A. Vincent and M. Meneguzzi, "The spatial structure and statistical properties of homogeneous turbulence," J. Fluid Mech. 225, 114501 (1991).

${ }^{26}$ C. Cichowlas, P. Bonaiti, F. Debbasch, and M.-E. Brachet, "Effective dissipation and turbulence in truncated Euler flows," Phys. Rev. Lett. 95, 264502 (2005).

${ }^{27}$ A. Pumir, "A numerical study of pressure fluctuations in three-dimensional, incompressible, homogeneous, isotropic turbulence," Phys. Fluids 666, 2071 (1994).

${ }^{28}$ A. Naso and F. S. Godeferd, "Statistics of the perceived velocity gradient tensor in a rotating turbulent flow," New J. Phys. 14, 125002 (2012).

${ }^{29}$ O. Zeman, "A note on the spectra and decay of rotating homogeneous turbulence," Phys. Fluids 666, 3221 (1994).

${ }^{30}$ P. D. Mininni, D. Rosenberg, and A. Pouquet, "Isotropization at small scales of rotating helically driven turbulence," J. Fluid Mech. 699, 263 (2012)

31 A. Delache, C. Cambon, and F. Godeferd, "Scale by scale anisotropy in freely decaying rotating turbulence," Phys. Fluids 26, 025104 (2014).

${ }^{32}$ M. Chertkov, A. Pumir, and B. I. Shraiman, "Lagrangian tetrad dynamics and the phenomenology of turbulence," Phys. Fluids 112, 2394 (1999).

${ }^{33}$ A. Naso and A. Pumir, "Scale dependence of the coarse-grained velocity derivative tensor structure in turbulence," Phys. Rev. E 72, 056318 (2005).

${ }^{34}$ A. Pumir and A. Naso, "Statistical properties of the coarse-grained velocity gradient tensor in turbulence: Monte-Carlo simulations of the tetrad model," New J. Phys. 125, 123024 (2010).

${ }^{35}$ L. Bourouiba and P. Bartello, "The intermediate Rossby number range and two-dimensional-three-dimensional transfers in rotating decaying homogeneous turbulence," J. Fluid Mech. 587, 139-161 (2007).

${ }^{36}$ S. B. Pope, Turbulent Flows (Cambridge University Press, 2000).

37 P. Sagaut and C. Cambon, Homogeneous Turbulence Dynamics (Cambridge University Press, 2008).

${ }^{38}$ M. Farge, G. Pellegrino, and K. Schneider, "Coherent vortex extraction in 3D turbulent flows using orthogonal wavelets," Phys. Rev. Lett. 87, 054501 (2001). 